\title{
Device Design and Scalability of an Impact Ionization MOS Transistor with an Elevated Impact Ionization Region
}

\author{
Eng-Huat Toh, Grace Huiqi Wang, Lap Chan, Ganesh Samudra, and Yee-Chia Yeo. \\ Silicon Nano Device Lab., Dept. of Electrical and Computer Engineering, \\ National University of Singapore (NUS), Singapore 117576. \\ Phone: +65 6516-2298, Fax: +65 6779-1103, Email: yeo@ieee.org
}

\begin{abstract}
This paper reports a novel L-shaped Impact-ionization MOS (LI-MOS) transistor structure that achieves a subthreshold swing of well below $60 \mathrm{mV} /$ decade at room temperature and operates at a low supply voltage. The device features an L-shaped or elevated Impact-ionization region (I-region) which displaces the hot carrier activity away from the gate dielectric region to improve hot carrier reliability and $V_{T}$ stability problems. Device physics and design principles for the LI-MOS transistor are detailed through extensive two-dimensional device simulations. The LI-MOS transistor exhibits excellent scalability, making it suitable for augmenting the performance of standard CMOS transistors in future technology generations.
\end{abstract}

\section{Introduction}

CMOS device scaling faces formidable challenges. Continuous reduction of $V_{T}$ with device scaling results in higher subthreshold leakage current $I_{\text {off }}$ and static power consumption, and is limited by the non-scalability of the subthreshold swing $S$. To realize $S$ of less than $60 \mathrm{mV} /$ decade at room temperature, a device structure [1] that modulates the breakdown voltage of a gated p-i-n structure to switch between the off and on states was proposed. The Impact-ionization MOS (I-MOS) device exploits the avalanche effect of impact ionization to realize a steep increase of current from the off-state to the on-state. An alternative device structure, the L-shaped I-MOS (LIMOS) [2] that uses elevated or L-shaped Impact-ionization region (I-region) relocates the impact ionization activity away from the gate dielectric region, thus reducing $V_{T}$ instability due to hot carriers. A selective epitaxial growth (SEG) which forms the elevated I-region allows accurate dopant profile engineering and easy controllability and scalability of the I-region dimensions. In addition, the LI-MOS device has a compact self-aligned device structure to improve packing density and is also CMOSprocess-compatible. This paper reports insights into the device physics and key device design principles of the LI-MOS transistor, and explores its scalability for future technology generations.

\section{Device Structure and Physics}

The LI-MOS transistor has a L-shaped I-region comprising an elevated I-region with thickness $T_{I}$ and resistance $R_{V}$, and a horizontal I-region with length $L_{S}$ and resistance 
$R_{H}$ (Fig. 1(a)). The L-shaped I-region has a total length $L_{I}=T_{I}+L_{S}$. The horizontal portion of the I-region has a p-type doping concentration $N_{B}$, and the elevated I-region has a doping concentration of $1 \times 10^{13} \mathrm{~cm}^{-3}$. The doping concentrations in the $\mathrm{n}^{+}$drain and $\mathrm{p}^{+}$source are $2 \times 10^{20} \mathrm{~cm}^{-3}$. Synopsys TCAD tools were used to study the device physics and design principles. Self-consistent impact ionization model [3] and bandto-band tunneling model were used with other essential models to accurately simulate sub-100 nm gate length devices at $300 \mathrm{~K}$.

I-MOS Transistor with

Elevated Impact-Ionization Region

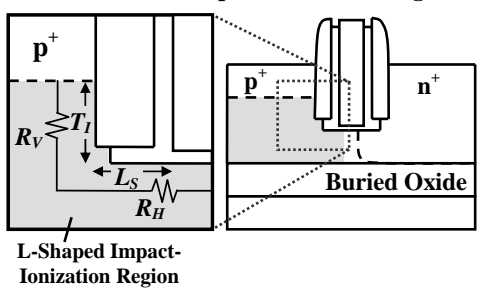

(a)

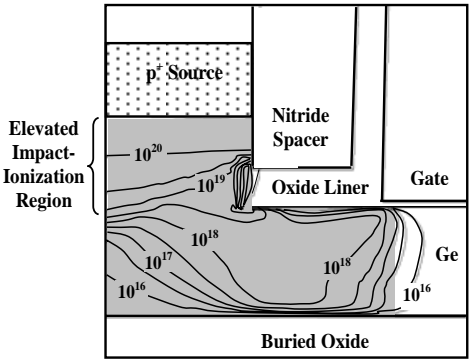

(b)

Fig. 1 (a) Schematic of an n-channel I-MOS Transistor with an Elevated Impact-ionization region (I-region), showing the equivalent resistances and dimensions in the I-region. (b) The concentration contour plot of holes generated by impact ionization for a Ge LI-MOS is shown. The majority of the holes generated are confined to the I-region (Gray).

The I-MOS transistor operates in the two states. In the off-state, the source is at a negative bias with the drain at a positive bias. Hence, the off-state current is the drift of the minority electron carriers. In the on-state, the gate induces sufficient band bending in the I-region to cause impact-ionization. Fig. 1(b) depicts the concentration contours of holes generated by impact-ionization in a Ge LI-MOS. By altering the resistance values of $R_{V}$ and $R_{H}$ (i.e. $N_{B}, T_{I}$, and $L_{S}$ ), hot carrier reliability could be improved by displacing the impact ionization activity away from the gate dielectric region. For instance, increasing $N_{B}$ causes more voltage drop across $R_{V}$ and increases the electric field in the elevated I-region. As a result, hot carrier activity is reduced in the horizontal I-region, and most of the hot carriers are confined in the elevated Iregion. In this study, the LI-MOS device is formed on a Germanium-on-Insulator (GOI) substrate to exploit the high impact ionization coefficient of Ge. The benefits of Ge comes not only from lower breakdown voltage, but also much lower off-state current $I_{\text {off }}$ and higher on-state current $I_{\text {on }}$.

\section{Device Design and Optimization}

Fig. 2(a) shows the dependence of $I_{D}-V_{G}$ on $N_{B}$ and excellent subthreshold swings of $\sim 1 \mathrm{mV} /$ decade for Ge n-channel LI-MOS transistors. $V_{T}$ decreases with increasing $N_{B}$, as illustrated in Fig. 2(b) unlike in conventional MOS transistors. Increasing $N_{B}$ leads to a greater voltage drop across the elevated I-region, therefore leading to higher electric field, increased impact ionization, and reduced $V_{T}$. The depletion width and $R_{H}$ depend inversely on $N_{B}$ which causes $V_{T}$ to decrease. In addition, $I_{\text {off }}$ is found to be lower in devices with lower $V_{T}$ (higher $N_{B}$ ) than in devices with higher $V_{T}$ (lower $N_{B}$ ). See Fig. 3(a). This is explained by a well-known inverse proportionality of $I_{\text {off }}$ to $N_{B}$ 
for a p-i-n diode [4]. Unlike the lateral I-MOS [1] where changing the substrate doping $N_{B}$ affects the whole I-region directly, the intrinsic region could be maintained in the elevated I-region while optimizing $N_{B}$ for better breakdown characteristics in the LI-MOS. Fig. 3(b) shows that $V_{T}$ increases with increasing $T_{I}$, for various $L_{S}$ values. An effective way to control $V_{T}$ is through control of the thickness of the elevated I-region $T_{I}$ by SEG. The use of SEG automatically miniaturizes the LI-MOS as there is no area penalty for including the elevated I-region. $L_{S}$ may also be employed to tune $V_{T}$ over a wide range if needed. However, $I_{\text {off }}$ increases rapidly as $L_{I}$ becomes comparable to $L_{G}$ (Fig. 4(a)). Nevertheless, higher $N_{B}$ allows simultaneous realization of lower $V_{T}$ and lower $I_{\text {off }}$, enabling device operation at lower $V_{D D}$ and yet out-perform the $I_{\text {off }} I_{\text {on }}$ specifications in the International Technology Roadmap for Semiconductors (ITRS-2006) [5].

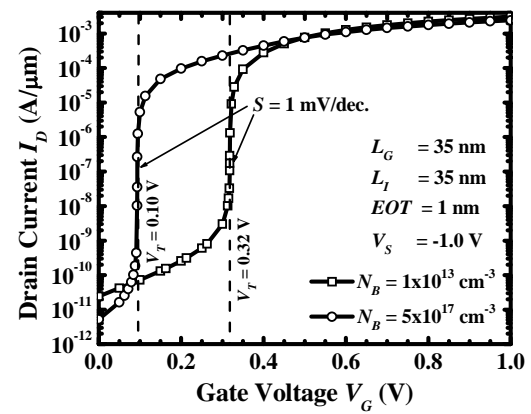

(a)

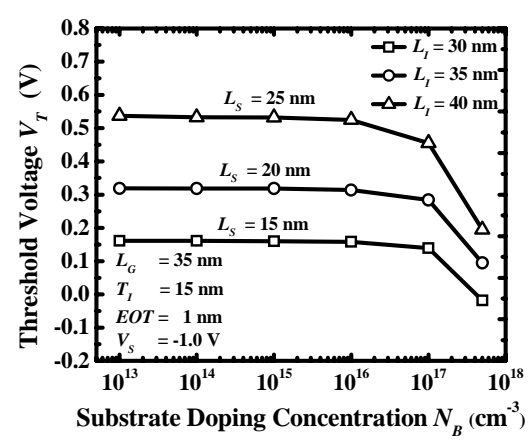

(b)

Fig. 2 (a) Simulated $I_{D}-V_{G}$ plot of Ge n-channel LI-MOS transistors with two different substrate doping concentrations $N_{B}$, showing a $1 \mathrm{mV} /$ decade transition between the off- and on- states. (b) Plot of threshold voltage $V_{T}$ versus substrate doping concentration $N_{B}$ for various length $L_{S}$, keeping $T_{I}$ fixed at $15 \mathrm{~nm}$. The L-shaped I-region has a total length $L_{I}=T_{I}+L_{S}$. A longer $L_{S}$ causes $V_{T}$ to drop faster as $N_{B}$ increases.

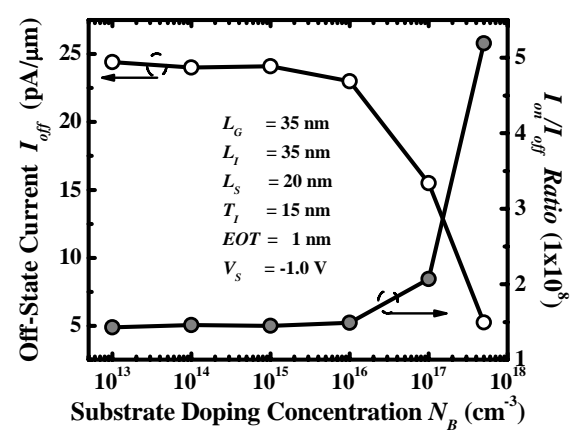

(a)

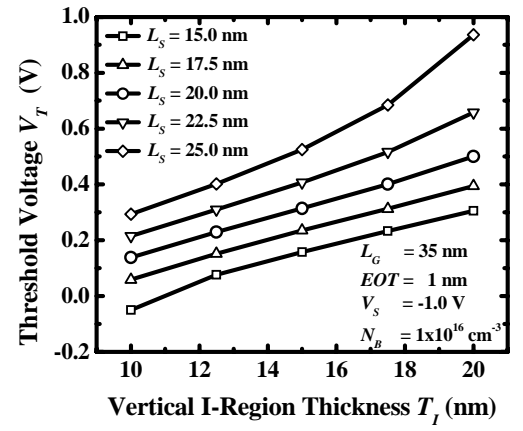

(b)

Fig. 3 (a) Plot of $I_{\text {off }}$ and $I_{\text {on }} / I_{\text {off }}$ ratio as a function of substrate doping concentrations $N_{B}$. $I_{\text {off }}$ decreases as $N_{B}$ increases. (b) Threshold voltage $V_{T}$ generally increases with increasing $T_{I} . L_{S}$ and $T_{I}$ are two adjustable parameters that allows for the flexibility of $V_{T}$ tuning. 


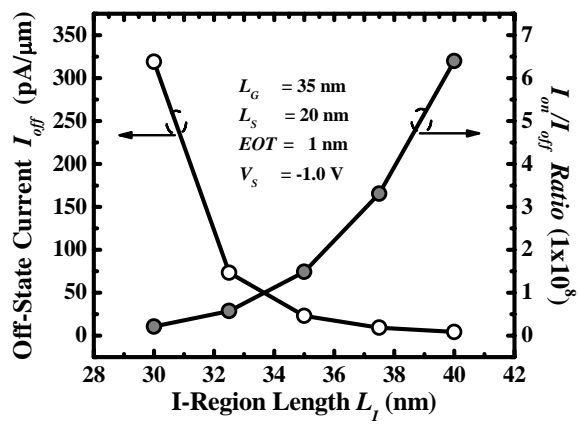

(a)

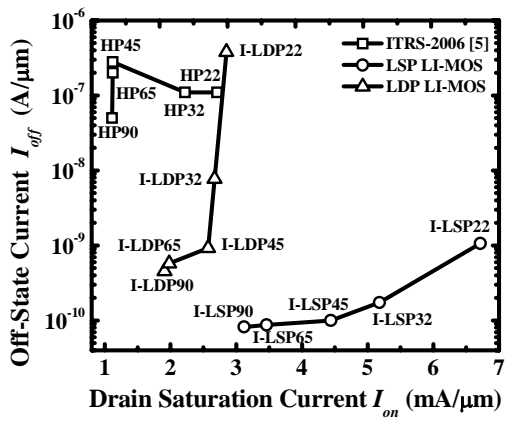

(b)

Fig. 4 (a) Plot of $I_{\text {off }}$ and $I_{\text {on }} / I_{\text {off }}$ ratio as a function of I-region length $L_{I}$. This also causes the $I_{\text {on }} / I_{\text {off }}$ ratio to decrease. (b) Superior capabilities of the Ge I-MOS technology using elevated Iregion, as compared with projected $I_{\text {off }}$ and $I_{\text {on }}$ targets of High-Performance (HP) CMOS technology nodes [5].

\section{Device Scalability}

To illustrate the scalability of the Ge LI-MOS transistor, simulations were carried out for each I-MOS technology node. An I-MOS technology node employs device structure parameters, e.g. gate length $L_{G}$, that are identical to those in the corresponding high-performance (HP) logic technology node [5], but $T_{I}$ and $L_{S}$ are used to optimize device performance. I-MOS transistors may be designed to achieve Low Static Power (LSP) or Low Dynamic Power (LDP) while maintaining high performance. I-MOS for LSP applications, i.e. I-LSP technology, may employ the same $V_{D D}$ as those in the corresponding HP CMOS technology node. Fig. 4(b) shows that I-LSP transistors demonstrate superb performance (lower $I_{\text {off }}$ and higher $I_{\text {on }}$ ) that exceeds the ITRS-2006 projections. I-MOS for LDP applications, i.e. I-LDP technology, may employ aggressively reduced $V_{D D}$ while satisfying the $I_{o n}$ specifications in ITRS-2006 [5].

\section{Conclusion}

The Ge LI-MOS, or I-MOS with an elevated impact-ionization region, is a CMOSprocess-compatible technology that is promising for augmenting the performance of conventional CMOS transistors. Its L-shaped impact-ionization structure allows the diversion of hot carrier activity away from the gate dielectric region, a critical improvement over existing I-MOS structures. The Ge LI-MOS device has excellent scalability in future technology nodes, enabling very significant reduction of power consumption in high-performance logic applications.

\section{References}

[1] K. Gopalakrishnan et al., IEDM Tech. Dig., pp. 289-292, 2002.

[2] E.-H. Toh et al., IEDM Tech. Dig., pp. 971-974, 2005.

[3] S. Selberherr, Springer-Verlag, 1984, ISBN 03878006.

[4] B. J. Baliga, J. Wiley, pp. 407-419, 1987, ISBN 0471819867.

[5] ITRS 2006, http://www.itrs.net/ 